\title{
Treating advanced non-small-cell lung cancer in Chinese patients: focus on icotinib
}

This article was published in the following Dove Press journal:

OncoTargets and Therapy

16 May 2014

Number of times this article has been viewed

\section{Jun-Li Liang' \\ Xiao-Cang Ren ${ }^{2}$ \\ Qiang Lin ${ }^{2}$}

'Department of Radiation Oncology, Hebei Medical University Fourth

Hospital, Shijiazhuang, People's Republic of China; ${ }^{2}$ Department of Oncology, North China Petroleum Bureau General Hospital of Hebei Medical University, Renqiu, Hebei Province, People's Republic of China

\begin{abstract}
Icotinib hydrochloride is an orally administered small-molecule reversible tyrosine kinase inhibitor that has been independently researched and developed and has independent intellectual property rights in the People's Republic of China. Clinical trials have demonstrated that the response to icotinib among advanced non-small-cell lung cancer (NSCLC) patients who received at least one platinum-based chemotherapy regimen was not inferior to gefitinib. Since being launched August 2011 in the People's Republic of China, icotinib has been widely used in clinics, and has become an important treatment option for Chinese patients with advanced NSCLC. The present study presents the Phase I, II, and III clinical trials of icotinib and discusses current clinical applications in the People's Republic of China and future research directions.
\end{abstract}

Keywords: targeted therapy, EGFR-TKI, NSCLC

\section{Introduction}

Among carcinomas, lung cancer has the highest global incidence and mortality. ${ }^{1}$ With increased industrialization, the incidence of lung cancer in the People's Republic of China is rising continuously. ${ }^{2}$ As one of the three classic therapeutic strategies, chemotherapy has exhibited a limited effect in the treatment of non-small cell lung cancer (NSCLC). In addition to more severe adverse effects, chemotherapy significantly affects patients' quality of life. Epidermal growth factor receptor (EGFR) tyrosine kinase inhibitor (TKI) drugs have a higher efficiency and lower toxicity and have played an important role in NSCLC. ${ }^{3}$

Icotinib hydrochloride is an orally administered small-molecule reversible TKI that has been independently researched and developed and has independent intellectual property rights in the People's Republic of China. Icotinib hydrochloride is a potent and specific EGFR-TKI, with an $\mathrm{IC}_{50}$ (half maximal inhibitory concentration) of $5 \mathrm{nM},{ }^{4}$ and a recent study has reported stronger activities than AstraZeneca and FDA. ${ }^{5}$ The antitumor mechanisms of icotinib may be related to the downregulation of Akt signal transduction. ${ }^{6}$ In-vitro experiments have suggested that the antitumor effects of icotinib against lung cancer cell lines with EGFR mutations are similar to those of gefitinib. ${ }^{7}$ In-vivo experiments have demonstrated that icotinib exerts anti-tumor effects against various xenograft tumors in nude mice, with a potential dose-effect relationship. ${ }^{8}$

In the present study, we summarize the Phase I, II, and III clinical trials of icotinib and discuss its current clinical application in the People's Republic of China and future research directions. 


\section{Clinical trial}

\section{Healthy subjects}

Seventy-five healthy Chinese male volunteers were randomly assigned to ten groups with ascending doses; the results reveal that no significant adverse effects were observed within the safety dose range (25-1,025 mg) of icotinib. ${ }^{5}$ A single-dose pharmacokinetic study with 100,350 , and $600 \mathrm{mg}$ threedose groups indicated that clinical and laboratory abnormalities are not correlated with dosage. In healthy subjects, the median peak time was $0.75-3.50$ hours, and the mean elimination half-life was $6.01-7.83$ hours. In addition, linear absorption characteristics were observed for $100-600 \mathrm{mg}$ dosages. Single-dose icotinib tablets (100-600 mg) were well tolerated, with only four adverse events occurring in three subjects. These adverse events were mild and transient, and the patients recovered quickly. Taking icotinib with a meal facilitates drug absorption. ${ }^{9}$ The maximum tolerated single administered dosage was not reached in the current trial, suggesting that there is great potential to increase the icotinib dosage.

\section{Advanced lung cancer Phase I/lla study}

The present study included two independent Phase I/IIa clinical trials performed at two different research centers. For the method of administration, one group was administered the drug three times daily (tid), ${ }^{10}$ while the other group was administered the drug twice daily (bid). ${ }^{11}$

In the icotinib tid trials, ${ }^{10}$ four incremental doses $(75,100$, 125 , and $150 \mathrm{mg}$ ) were used. The results indicated that the patients in each group tolerated the drug well; level III or higher toxicities were not observed in any group, suggesting that the maximum tolerated dose was not reached. Even $1,025 \mathrm{mg} /$ day can be well tolerated by healthy subjects, and partial response in the patients was observed with $300 \mathrm{mg}$ /day (100 mg, tid), suggesting a significant antitumor effect at this dosage level. Therefore, it was not necessary to increase the dosage further. Pharmacokinetic studies indicated that a single oral dose of icotinib can be rapidly absorbed by patients, with a peak time of 2 hours and a half-life of 6 hours, which was consistent with the results obtained from healthy volunteers. ${ }^{9}$

In the icotinib bid administration group ${ }^{11}$ four patients exhibited grade III-V toxicity, including two deaths. One death was confirmed to be disease related rather than drug related after autopsy. The other death was confirmed to be due to both disease progression and drug toxicity according to biopsy. In the $150 \mathrm{mg}$ bid group, one case developed pancreatitis, which was considered to be non-drug related, and one case of grade I nausea and upper abdominal pain was judged as drug related.

According to the results from these two studies, it is recognized that the tid administration was better tolerated and that the drug elimination half-life was 6 hours. Based on the findings from the Phase I trial, researchers expanded the enrollment for the $100 \mathrm{mg}$ (tid), $125 \mathrm{mg}$ (tid), and $150 \mathrm{mg}$ (tid) groups and further evaluated the efficacy and safety in a Phase IIa clinical trial. ${ }^{5}$

A total of 103 patients were enrolled in the Phase I/IIa study, with the following enrollment criteria: histologically or cytologically confirmed diagnosis of IIIB or IV NSCLC and a history of one or more prior ineffective platinum-based chemotherapy treatments. The study results indicated that icotinib had a favorable safety profile, with the most common adverse reactions being rash and diarrhea (both were grade I or II), with incidence rates of $34 \%(36 / 103)$ and $11 \%(12 / 103)$, respectively. Only three patients exhibited a grade III or above rash. Most cases of rash or diarrhea occurred after 1 week of icotinib treatment, were transient, and only lasted for 2-3 weeks, and there was no need to terminate icotinib or additional clinical intervention. Very few patients exhibited increased transaminase activity, and these were also transient and did not require the termination of icotinib or additional clinical intervention. The use of icotinib resulted in significant antitumor effects: the disease control rate (DCR) was 78.1\%, the objective response rate (ORR) was $29.2 \%$, and three patients experienced complete remission. ${ }^{5,10,11}$

The above Phase I/IIa study demonstrated that icotinib had significant efficacy and a satisfactory safety profile for advanced NSCLC treatment, and it was recommended for Phase III clinical trials at a dose of $125 \mathrm{mg}$, tid, oral administration for 28 days.

\section{Phase III trial}

The icotinib Phase III trial icotinib versus gefitinib in previously treated advanced non-small-cell lung cancer (ICOGEN) was implemented in 27 research centers in nine cities in the People's Republic of China and was a randomized, doubleblind, head-to-head, multicenter study. ${ }^{12}$ This Phase III trial evaluated the efficacy and safety of icotinib and gefitinib in treating locally advanced or metastatic progressive NSCLC patients who previously received at least one platinum-based chemotherapy treatment. The study was designed as a noninferiority trial, with the primary endpoint being progressionfree survival (PFS) and the secondary endpoints including overall survival (OS), (ORR), DCR, time to progression (TTP), quality of life, and safety and tolerability. A total of 
400 cases were initially enrolled in the study, and one case was later excluded from the study. The remaining 399 patients were assigned at a 1:1 ratio to the icotinib group (200 cases) and the gefitinib group (199 cases). The two groups were balanced with regard to baseline conditions, with 396 cases being included in the full analysis set.

In terms of the primary endpoint, icotinib was comparable to gefitinib in terms of PFS, with a median PFS of 4.6 and 3.4 months (hazard ratio $[\mathrm{HR}]=0.84, P=0.13$ ), respectively. Icotinib increased PFS by 1.2 months compared with gefitinib. No difference in TTP was observed between the icotinib and gefitinib groups, although a slightly prolonged TTP was exhibited by the icotinib group compared with the gefitinib group, at 5.2 and 3.7 months $(\mathrm{HR}=0.78, P=0.065)$, respectively. The OS-related data collection is still in progress; there is no difference in the median OS, which was 13.3 and 13.9 months, respectively $(\mathrm{HR}=1.02, P=0.57)$. The ORR and DCR of the icotinib and gefitinib groups were $27.6 \%$ and $27.2 \%$ versus $75.4 \%(150 / 199)$ and $74.9 \%(146 / 196)$, respectively, with no significant differences between the two groups $(P=0.91,0.90)$. Notably, subgroup analysis revealed that for 96 cases with non-squamous cell carcinoma, PFS was significantly higher in the icotinib group than in the gefitinib group ( $\mathrm{HR}=0.61$ (0.39-0.97), $P=0.035)$; among the squamous cell carcinoma cases, the ORR in the icotinib group was also significantly higher than that in the gefitinib group, with values of $15 \%$ $(5 / 34)$ and $6 \%(2 / 36)$, respectively. These results must be verified in subsequent studies.

In terms of safety, the percentage of overall drug-related adverse events was significantly lower in the icotinib group compared with the gefitinib group, with values of $61 \%(121 / 200)$ and $70 \%(140 / 199)$, respectively, $P=0.045$. Further analysis revealed that the incidence of diarrhea in the icotinib group was significantly lower than that in the gefitinib group ( $19 \%$ versus $28 \%, P=0.033)$. The improvement in the overall adverse reactions was primarily due to less incidence of diarrhea. Except for vomiting (ten cases versus nine cases), the incidences of all the other drugrelated adverse reactions in the icotinib group were lower than those in the gefitinib group.

In the 399 cases in the present study, a total of 152 cases were subjected to specimen collection, of which $34 \%$ (134 cases) received EGFR test results. The overall EGFR mutation rate for the two groups was $51 \%(68 / 134)$. Out of the 68 cases in the icotinib group, EGFR mutations occurred in 29 (43\%); out of the 66 cases from the gefitinib group, EGFR mutations occurred in 39 (59\%). No significant difference $(P=0.72)$ in mutations was observed between the two groups. The mutants 19Del (63\%) and Leu858Arg (29\%) accounted for $89 \%$ of the mutations. PFS for patients with EGFR mutations was significantly higher than for those with wild-type EGFR: median PFS, 6.2 months versus 2.3 months (HR $=0.44, P<0.0001$ ), while the PFS and OS results for cases with EGFR mutations were not significantly different between the icotinib and gefitinib groups.

In summary, the ICOGEN study demonstrated that in terms of the primary endpoint of PFS, icotinib was comparable to gefitinib. In terms of the secondary endpoints such as OS, TTP, ORR, and DCR, both drugs could significantly improve the patients' quality of life to a similar extent. In terms of the incidence of adverse reactions, icotinib was significantly better than gefitinib, which indicated the increased safety of icotinib.

To further confirm the efficacy and safety of icotinib, as part of the ICOGEN studies, a single-arm study expanded the sample size for the icotinib group in 15 centers in the People's Republic of China. A total of 128 cases were enrolled, and 124 cases were included in the full analysis set. The results were as follows: ORR $=25.8 \%, \mathrm{DCR}=67.7 \%$, $\mathrm{PFS}=5.0$ months, TTP $=5.4$ months, and OS =17.6 months, and rash and diarrhea rates were $17.1 \%$ and $25.5 \%$, respectively. Compared with the Phase III randomized trial, this single-arm study showed comparable (or better) efficacy and less adverse reactions, which further supported the role of icotinib in treating NSCLC patients who had received prior ineffective treatment. ${ }^{13}$

\section{Current status of clinical application}

Icotinib is a self-developed EGFR-TKI inhibitor from the People's Republic of China, and Phase I, II, and III clinical data for icotinib were obtained from the Chinese population. Its price is approximately $70 \%$ of that of gefitinib and $60 \%$ of that of erlotinib. Icotinib has the advantages of having less toxicity compared with chemotherapy, being convenient to use and having no requirement for hospitalization. In addition, one investigation has demonstrated the pharmacoeconomic benefits of icotinib compared with traditional second-line chemotherapy. ${ }^{14}$ Thus, icotinib has been widely used in clinics in the People's Republic of China. Icotinib has been primarily used in treating advanced NSCLC patients for second-line or above therapy after progression of first-line treatment. Icotinib has also been used to treat patients who are not suitable for chemotherapy due to physical conditions or who refuse to receive chemotherapy as a first-line treatment. Because icotinib has only been available for 2 years and 6 months, only a limited number of studies on this 
drug have been published in Chinese in the People's Republic of China, of which most were retrospective. Nevertheless, relatively complete data are available and are described in this article.

\section{Second-line and above}

Table 1 lists nine studies for icotinib as a second-line or above treatment. ${ }^{15-23}$ The number of enrolled cases ranged from 30 to 149. Except in He et al, ${ }^{23}$ the majority of patients had adenocarcinoma (69.1\%-100\%). Only two of the studies were prospective studies, ${ }^{15,21}$ the enrollment criteria were similar to that of the ICOGEN, and favorable results were obtained (except in He et $\mathrm{al}^{23}$ ): OR 22.1\%-76.3\% and DCR 62.7\%-100\%. Seven studies reported that PFS was 5.0-10.0 months, ${ }^{17-23}$ and PFS in patients with a mutant EGFR was significantly superior to those with wild-type EGFR. ${ }^{17,18}$ Four studies reported that OS was 12.3-15.1 months. ${ }^{20-23}$ The largest number of cases was studied by Shao et al, ${ }^{20}$ whose results included ORR of $22.1 \%$, DCR of $71.8 \%$, PFS of 5.03 months, and OS of 12.3 months, which were similar to the ICOGEN data of $27.6 \%, 75.4 \%, 4.6$ months, and 13.3 months, respectively. $\mathrm{Su}$ et $\mathrm{al}^{19}$ reported the results of NSCLC cases with mutations, which showed the best efficacy: ORR 76.3\%, DCR $100 \%$, and PFS 10 months. Zhang et al $^{18}$ studied 31 cases of NSCLC with brain metastases (77.4\% were adenocarcinoma, 25 cases received cephalic radiotherapy) and reported an ORR of $25.8 \%$ for brain lesions and $38.7 \%$ for systemic lesions, and a DCR of $83.9 \%$ for brain lesions and $87.1 \%$ for systemic lesions, with a PFS of 6.5 months. Despite being a retrospective study with a small sample size, the study by Zhang et al ${ }^{18}$ suggested that icotinib has promising efficacy for lung cancer with brain metastasis.

He et $\mathrm{al}^{23}$ performed a retrospective analysis of 31 patients with stage IV squamous cell carcinoma (nine cases as a second-line treatment, 22 cases as a second-line or above treatment) and reported the following results: ORR, 6.5\%; DCR, 38.7\%; and PFS, 2.1 months. These were similar to the results reported in the literature. ${ }^{24-26}$ These findings indicate that for advanced squamous cell carcinoma patients who have received prior ineffective first-line treatment and cannot tolerate or refused to receive secondary chemotherapy, icotinib might be an alternative option.

\section{First-line, second-line, and third-line and above therapy}

Table 2 lists ten retrospective studies, ${ }^{27-36}$ each containing first-, second-, and third-line and above therapies; the number of enrolled cases in each study was 17-89. The patients mainly had adenocarcinoma, which accounted for $78.3 \%-100 \%$ of the cases. The following promising results were obtained: RR, 26.2\%-56.7\%; and DCR, 69.6\%-88.2\%. Three studies reported PFS of 3.8, 7.0, and 8.5 months, ${ }^{27,35,36}$ and the PFS from patients with mutations was significantly superior to those with the wild-type. ${ }^{27}$

Song et $\mathrm{al}^{27}$ performed EGFR tests for all the cases enrolled in their study and observed that the mutation rate was $73.5 \%$ (36/49), with 19 cases receiving first-line treatment and 17 cases receiving second-line or above treatment; the PFS was 9.5 and 8.5 months, respectively, with no significant differences $(P=0.41)$. In patients with positive mutations, the PFS was 8.7 months for the 12 patients with brain metastases and 10.0 months for the 24 patients without brain metastases; no significant differences were observed between the two groups $(P=0.69)$, suggesting that icotinib is effective for the mutant type with brain metastases. The median OS was not reached in the mutation group. However, the median OS was 12.6 months for the wild-type, with a significant difference $(P<0.001)$ between the two groups. ${ }^{27}$ Eight studies compared the short-term efficacy of icotinib as a first- or second-line or above treatment; four studies reported that

Table I Icotinib in second-line therapy and above

\begin{tabular}{|c|c|c|c|c|c|c|c|c|c|}
\hline Study & $\begin{array}{l}\text { Number } \\
\text { of Cases }\end{array}$ & $\begin{array}{l}\text { Proportion of } \\
\text { adenocarcinoma }\end{array}$ & OR & DCR & $\begin{array}{l}\text { PFS, } \\
\text { months }\end{array}$ & $\begin{array}{l}\text { OS, } \\
\text { months }\end{array}$ & Rash & Diarrhea & $\begin{array}{l}\text { Dysfunction } \\
\text { of liver }\end{array}$ \\
\hline Li et al $^{15}$ & 30 & $90 \%$ & $46.7 \%$ & $73.3 \%$ & - & - & $43.3 \%$ & $23.3 \%$ & $10 \%$ \\
\hline Chen et $\mathrm{al}^{16}$ & 67 & $91 \%$ & $38.8 \%$ & $62.7 \%$ & - & - & $26.8 \%$ & $13.4 \%$ & $10.4 \%$ \\
\hline Nong et $\mathrm{al}^{17}$ & 60 & $90 \%$ & $45 \%$ & $80 \%$ & 6.7 & - & $38.3 \%$ & $14.2 \%$ & $1.7 \%$ \\
\hline Zhang et $\mathrm{al}^{18}$ & 31 & $77.4 \%$ & $38.7 \%$ & $87.1 \%$ & 6.5 & - & $22.6 \%$ & $12.9 \%$ & $3.2 \%$ \\
\hline Su et al $^{19}$ & 38 & $94.7 \%$ & $76.3 \%$ & $100 \%$ & 10 & - & $63.2 \%$ & $28.9 \%$ & $10.5 \%$ \\
\hline Shao et $\mathrm{al}^{20}$ & 149 & $69.1 \%$ & $22.1 \%$ & $71.8 \%$ & 5.03 & 12.3 & $40.9 \%$ & $18.1 \%$ & $7.4 \%$ \\
\hline Zhang et $\mathrm{al}^{21}$ & 40 & $85 \%$ & $25 \%$ & $70 \%$ & 5.3 & 15.1 & $25 \%$ & $7.5 \%$ & - \\
\hline Zhang et $\mathrm{al}^{22}$ & 51 & $100 \%$ & $23.5 \%$ & $76.5 \%$ & 5.1 & 12.5 & $29.4 \%$ & $17.6 \%$ & - \\
\hline $\mathrm{He}$ et $\mathrm{al}^{23}$ & 31 & $0 \% *$ & $6.5 \%$ & $38.7 \%$ & 2.1 & 21.8 & $38.7 \%$ & $16.1 \%$ & $6.5 \%$ \\
\hline
\end{tabular}

Notes: “-”, not available; *all cases were squamous cell carcinoma.

Abbreviations: DCR, disease control rate; OS, overall survival; PFS, progression-free survival. 
Table 2 Icotinib in first-, second-, and third-line therapy and above

\begin{tabular}{|c|c|c|c|c|c|c|c|c|c|c|}
\hline Study & $\begin{array}{l}\text { Number } \\
\text { of Cases }\end{array}$ & $\begin{array}{l}\text { Proportion of } \\
\text { first-line therapy }\end{array}$ & OR & DCR & $\begin{array}{l}\text { RR/DCR in first- } \\
\text { line therapy }\end{array}$ & $\begin{array}{l}\text { PFS, } \\
\text { months }\end{array}$ & $\begin{array}{l}\text { OS, } \\
\text { months }\end{array}$ & Rash & Diarrhea & $\begin{array}{l}\text { Dysfunction } \\
\text { of liver }\end{array}$ \\
\hline Song et $\mathrm{a}^{27}$ & 49 & $38.8 \%$ & $38.7 \%$ & $77.4 \%$ & - & 8.5 & - & $38.8 \%$ & $24.5 \%$ & - \\
\hline Gu et $\mathrm{a}^{28}$ & 89 & $19.1 \%$ & $36 \%$ & $69.7 \%$ & - & - & - & $33.7 \%$ & $16.9 \%$ & $5.6 \%$ \\
\hline Sun 29 & 23 & $39 \%$ & $39.1 \%$ & $69.6 \%$ & $66.7 \% * / 77.8 \%$ & - & - & $8.7 \%$ & $8.7 \%$ & $0 \%$ \\
\hline Zeng et $\mathrm{a}^{30}$ & 67 & $23.9 \%$ & $35.8 \%$ & $74.6 \%$ & $56.3 \% * / 93.8 \%$ & - & - & $20.9 \%$ & $11.9 \%$ & $1.5 \%$ \\
\hline Zhang et $\mathrm{al}^{31}$ & 50 & $44 \%$ & $44 \%$ & $82 \%$ & $63.6 \% * / 78.6 \%$ & - & - & $28 \%$ & $16 \%$ & $4 \%$ \\
\hline Sun et $\mathrm{al}^{32}$ & 17 & $52.9 \%$ & $52.9 \%$ & $88.2 \%$ & $66.7 \% * / 88.2 \%$ & - & - & $11.8 \%$ & $11.8 \%$ & $0 \%$ \\
\hline Li et $a^{133}$ & 59 & $33.9 \%$ & $45.8 \%$ & $74.6 \%$ & $40 \% / 75 \%$ & - & - & $35.6 \%$ & $15.3 \%$ & $5.1 \%$ \\
\hline Yu et $\mathrm{al}^{34}$ & 30 & $33.7 \%$ & $56.7 \%$ & $80 \%$ & $63.5 \% / 72.7 \%$ & - & - & $60 \%$ & $33.3 \%$ & $16.7 \%$ \\
\hline Wu et $\mathrm{al}^{35}$ & 42 & $40.5 \%$ & $26.2 \%$ & $78.6 \%$ & $29.4 \% / 82.4 \%$ & 3.8 & 5.2 & $23.8 \%$ & $9.5 \%$ & $7.1 \%$ \\
\hline Wang et $\mathrm{a}^{36}$ & 66 & $51.5 \%$ & $38.7 \%$ & $77.4 \%$ & $50 \% / 86.7 \%$ & 7 & - & $40.9 \%$ & $18.2 \%$ & $1.5 \%$ \\
\hline
\end{tabular}

Notes: “-”, not available; *ORR in first-line therapy was significantly higher than that in second-line therapy and above.

Abbreviations: DCR, disease control rate; OS, overall survival; PFS, progression-free survival.

the ORR was significantly higher when icotinib was used as a first-line rather than second-line or above treatment, ${ }^{29-32}$ and four other studies ${ }^{33-36}$ reported no differences between these groups. Because the numbers of enrollment in these ten retrospective studies were limited and the EGFR mutation detection rates were relatively low in all research groups, comparing the efficacy between the first-line and secondline and above groups requires further study because of the unknown mutation status in most cases.

The above eight studies ${ }^{29-36}$ reported the efficacy of icotinib as a first-line treatment: ORR was $29.4 \%-66.7 \%$ and DCR was $75 \%-93.8 \%$, which were higher than the ORR and DCR of icotinib as the second-line and above treatment, which were $21.4 \%-52.6 \%$ and $56.3 \%-85.7 \%$, respectively. In addition, the ORR and DCR results were not less than the ICOGEN results (ORR was $27.6 \%$ and DCR was $75.4 \%$ ), ${ }^{12}$ which demonstrated that icotinib might be non-inferior as a first-line therapy to second-line and above therapy. The efficacies obtained from these eight studies using icotinib as a second-line and above treatment were ORR $21.4 \%-52.6 \%$ and DCR $56.3 \%-85.7 \%$, which were consistent with the nine studies with only enrolled cases using icotinib as a secondline and above treatment (RR was $22.1 \%-76.3 \%$, DCR was $62.7 \%-100 \%){ }^{15-23}$

One study ${ }^{34}$ included six advanced cases using gefitinib treatment and resulted in two cases of partial response and two cases of stable disease (SD). Zeng et al ${ }^{30}$ studied 14 cases with prior application of EGFR-TKIs (three cases of erlotinib and 11 cases of gefitinib) and observed that RR was $14.3 \%$ and DCR was $57.1 \%$, suggesting the potential effectiveness of icotinib following resistance to other EGFR-TKIs.

\section{The first-line treatment}

Table 3 lists three small sample retrospective studies using icotinib as the first-line treatment for NSCLC. ${ }^{37-39}$ Yang et $\mathrm{al}^{37}$ analyzed the preliminary results of 56 cases of lung adenocarcinoma using icotinib as the first-line treatment and reported the following: $94.6 \%$ of patients were stage IV, 36 cases had unknown EGFR status, 20 cases received genetic screening, 18 cases had a mutation, ten cases had mutations at 19DEL, eight cases had mutations at L858R, overall ORR was $46.4 \%$, DCR was $78.6 \%$, and the 18 cases with mutations had ORR of $66.7 \%$ and DCR of $94.4 \%$. Icotinib could rapidly improve symptoms, and 31 patients (55.4\%) showed amelioration of symptoms after treatment. The improvement occurred 2-14 days following treatment, with a median effective day on day 10 after treatment. The short-term efficacy and efficacy with mutation were similar to the results obtained from the IPASS study ( $43.0 \%$ and $71.2 \%$ ), while the subgroup analysis indicated that the efficacy was related to the status of smoking, sensitive EGFR mutations, and rash, which were also consistent with the IPASS study. ${ }^{40}$

Table 3 Icotinib in first-line therapy

\begin{tabular}{|c|c|c|c|c|c|c|c|c|c|}
\hline Study & $\begin{array}{l}\text { Number } \\
\text { of Cases }\end{array}$ & $\begin{array}{l}\text { Proportion of } \\
\text { adenocarcinoma }\end{array}$ & OR & DCR & $\begin{array}{l}\text { PFS, } \\
\text { months }\end{array}$ & $\begin{array}{l}\text { OS, } \\
\text { months }\end{array}$ & Rash & Diarrhea & $\begin{array}{l}\text { Dysfunction } \\
\text { of liver }\end{array}$ \\
\hline Yang et $\mathrm{al}^{37}$ & 56 & $100 \%$ & $46.4 \%$ & $78.6 \%$ & - & - & $28.5 \%$ & $10.7 \%$ & $10.7 \%$ \\
\hline $\mathrm{He}$ et $\mathrm{al}^{38}$ & 21 & $76.2 \%$ & $42.9 \%$ & $85.7 \%$ & 7.2 & - & $33.3 \%$ & $33.3 \%$ & $9.5 \%$ \\
\hline Quan et $\mathrm{al}^{39}$ & 34 & $58.8 \%$ & - & $68.75 \% *$ & - & - & $23.5 \%$ & $20.6 \%$ & $2.9 \%$ \\
\hline
\end{tabular}

Notes: *RR of pleural effusion was 71.88\%; “-”, not available.

Abbreviations: DCR, disease control rate; OS, overall survival; PFS, progression-free survival. 
He et $\mathrm{al}^{38}$ reviewed 21 patients with stage IV NSCLC using icotinib as the first-line treatment and reported the following: the $E G F R$ mutation status was unknown, ORR was 42.9\%, DCR was $85.7 \%$, and PFS was 7.2 months; the ORR and PFS results were consistent with the IPASS results. ${ }^{40}$

Quan et $\mathrm{a}{ }^{39}$ reported 34 patients with advanced NSCLC and first symptoms of pleural effusion using icotinib as a first-line treatment and reported the following: ten cases received $E G F R$ tests, four cases had a mutation, six cases were wild-type, pleural effusion DCR was $71.9 \%$, and disease DCR was $68.8 \%$.

\section{Combination therapy}

Zheng et al ${ }^{41}$ reported preliminary results from 28 cases of stage IV lung adenocarcinoma with only brain or bone metastases and receiving simultaneous radiotherapy together with icotinib: 16 cases had EGFR mutations; 12 cases had unknown mutation status; icotinib was given $125 \mathrm{mg}$, tid; and simultaneous radiotherapy was used and implemented as the conventional fractionated irradiation strategy, including primary tumors in the lesion of lung ( $60 \mathrm{~Gy}$ ), the lesion of bone (46 Gy), or brain metastases (full cranial 40 Gy). The patients tolerated the treatment well, with mild adverse reactions. A satisfactory short-term curative effect was obtained as follows: the local control rate of bone metastases was $95 \%$ (19/20), the control rate of brain metastases was $70 \%(7 / 10)$, the primary lung tumor local control rate after 6 months was $82.1 \%(23 / 28)$, and the OS after 6 months was $82.1 \%$. Lv et $\mathrm{al}^{42}$ reported preliminary results from 11 cases with stage IIB-IIIA NSCLC after icotinib combination chemotherapy (gemcitabine + cisplatin) treatment: ORR was $45 \%$, and six cases accepted surgery without serious complications. Based on these findings, Lv et al ${ }^{42}$ suggested that further evaluation is required for this combination therapy.

\section{Toxicity}

Based on published studies, icotinib, both as a second-line or above treatment or as a first-line treatment, exerted favorable safety and tolerability; the vast majority of patients can tolerate treatment, and discontinuation of treatment due to adverse reactions only occurred in a few cases. ${ }^{36,39}$ Common adverse reactions were rash, diarrhea, and abnormal liver function, with incidence rates of $8.7 \%-63.2 \%, 8.7 \%-33.3 \%$, and $0.0 \%-14.1 \%$, respectively. Most rash and diarrhea cases were grade I/II; few reached grade III or above. Abnormal liver function was only at grade I/II. Only two cases of interstitial pneumonia were reported. ${ }^{20,39}$ Of these two cases, one discontinued treatment, ${ }^{39}$ while the other was grade I, with unknown information about continuing treatment. ${ }^{20}$ No treatment-related deaths were reported in any study.

Overall, two studies ${ }^{19,34}$ indicated that the incidence of rash was greater than $60 \%$, which appears to be much higher than the $41 \%$ reported by ICOGEN. These two studies were small sample retrospective studies (38 cases and 30 cases), and selection bias might have occurred; thus, the results are not representative. The $63.2 \%$ of rash occurred in all patients with EGFR mutations. ${ }^{19}$ The short-term efficacy in this study was higher than in other studies, with $73.2 \%$ of ORR and 10 months of PFS. Furthermore, rash occurrence was associated with efficacy; the high incidence of rash might be due to $100 \%$ of patients having EGFR mutations in this study, which might be consistent with IPASS. ${ }^{40}$

Table 4 compares the adverse reactions from two studies with a relatively large number of enrolled cases (149 cases and 89 cases $)^{20,28}$ and ICOGEN. The incidence and severity of rash, diarrhea, and abnormal liver function in these two studies were comparable with those from ICOGEN. The incidences in one study ${ }^{28}$ appeared to be slightly lower than those in ICOGEN, which might be because this study had $19.1 \%$ first-line treatment patients.

\section{Phase IV clinical data}

After icotinib was launched, a total of 5,549 copies of feedback forms with icotinib safety and efficacy information from 500 tumor-specializing hospitals or major general hospitals were collected from August 2011 to October 2012.43 The primary data are presented in Table 5. This large data sample showed that the preliminary results from the shortterm efficacy in the Phase IV trial were comparable with the Phase III results. Moreover, adverse reactions were also comparable or even better. Patients older than 70 years tolerated

Table 4 Comparison of side effects of icotinib

\begin{tabular}{|c|c|c|c|c|c|c|c|}
\hline \multirow[t]{2}{*}{ Study } & \multirow{2}{*}{$\begin{array}{l}\text { Number } \\
\text { of Cases }\end{array}$} & \multicolumn{2}{|l|}{ Rash } & \multicolumn{2}{|l|}{ Diarrhea } & \multicolumn{2}{|c|}{ Dysfunction of liver } \\
\hline & & Grade I/II & Grade III/IV & Grade I/II & Grade III/IV & Grade I/II & Grade III/IV \\
\hline Shi et al ${ }^{12}$ & 200 & $40.0 \%$ & $<1.0 \%$ & $19.0 \%$ & 0 & $11.0 \%$ & 0 \\
\hline Shao et $\mathrm{a}^{20}$ & 149 & $40.0 \%$ & $1.3 \%$ & $18.1 \%$ & $<1.0 \%$ & $7.4 \%$ & 0 \\
\hline Gu et $\mathrm{al}^{28}$ & 89 & $33.7 \%$ & 0 & $16.9 \%$ & 0 & $5.6 \%$ & 0 \\
\hline
\end{tabular}


Table 5 Comparisons between Phase 3 trial and Phase 4 trial of icotinib

\begin{tabular}{|c|c|c|c|c|c|c|c|c|}
\hline Study & Phase & Specific population & $\begin{array}{l}\text { Number } \\
\text { of Cases }\end{array}$ & ORR & DCR & Rash & Diarrhea & $\begin{array}{l}\text { Dysfunction } \\
\text { of liver }\end{array}$ \\
\hline Shi et al ${ }^{12}$ & III & All patients & 200 & $27.6 \%$ & $75.4 \%$ & $41 \%$ & $22 \%$ & $11 \%$ \\
\hline \multirow[t]{6}{*}{$\operatorname{Sun}^{43}$} & IV & All patients & 5,549 & $30 \%$ & $80.6 \%$ & $17.4 \%$ & $8.5 \%$ & $0.8 \%$ \\
\hline & & $>70$ years old & $|, 57|$ & - & - & $16.7 \%$ & $8.1 \%$ & - \\
\hline & & EGFR mutated & & & & & & \\
\hline & & First-line & 144 & $56.3 \% *$ & $95.2 \% *$ & - & - & - \\
\hline & & Second-line and above & 521 & $47.1 \%$ & $91.5 \%$ & - & - & - \\
\hline & & EGFR wild & 214 & $17.8 \%$ & $75.7 \%$ & - & - & - \\
\hline
\end{tabular}

Notes: *ORR and DCR in first-line therapy were significantly higher than those in second-line therapy and above in Phase IV trial; "-", not available. Abbreviations: DCR, disease control rate; EGFR, epidermal growth factor receptor; ORR, objective response rate.

the drug well. There were 879 cases with a confirmed $E G F R$ mutation status, and 665 cases had mutilations (75.7\%). First-line treatment patients with mutations exhibited better ORR and DCR than second-line and above treatment patients, which further supported that icotinib as a first-line treatment can be used in treating NSCLC patients with mutations. Icotinib also showed some effectiveness for NSCLC patients with wild-type EGFR (ORR was 17.8\% and DCR was 75.7\%). Professor Sun, one of the principal ICOGEN investigators, stated that targeted treatment with icotinib for NSCLC had been confirmed by the evidence from Phase IV trial. ${ }^{43}$

\section{Summary of the clinical application}

Icotinib has been widely applied in the People's Republic of China, with the majority of experiences obtained as a second-line and above treatment; the short-term efficacy is consistent with the results from the Phase III trial of ICOGEN. The Phase IV clinical data confirmed the safety of application on a large sample size. The preliminary efficacy was consistent with Phase III results and was also observed during first-line treatment. Icotinib may also be effective for the following conditions: brain metastases, dose increases following prior failure with routine dosages, and treatment after progression to other EGFR-TKI drugs. Due to the short follow-up time, its long-term survival must be further determined.

Currently, icotinib has been used on NSCLC as a first-, second-, and third-line or above treatment. However, there has been insufficient evidence for its use as a first-line treatment. Due to the short launching time of icotinib, most published clinical studies were small-sample, retrospective studies with short follow-up times and suffered from insufficient survival data, imprecise research methods, low EGFR mutation detection rates in most cases, and biased patient selection. The long-term efficacy of icotinib must be further confirmed with well-designed strategy, large sample collection, and multicenter clinical trials.

\section{Research directions}

There are several possible future clinical research directions for icotinib. First, use icotinib as a routine first-line treatment for patients with $E G F R$ mutations. Second, increase the dose of icotinib for patients who do not respond to routine dosage. $\mathrm{Li}$ et $\mathrm{al}^{44}$ reported the progress of one patient with a routine dose of icotinib, and the disease was only controlled with an increased dose. Third, combine icotinib with chemotherapy or radiotherapy. Fourth, because the Phase I study of icotinib did not reach dose-limiting toxicity, ${ }^{10}$ according to the results from healthy subjects in Phase I trials, the current recommended dose of $125 \mathrm{mg}$, tid, might be several folds away from the maximum tolerated dose, suggesting that the dose can be significantly increased. ${ }^{5}$ Doubling the dose or using even higher doses for brain metastasis, EGFR wild-type, and failure with prior gefitinib and erlotinib ${ }^{45}$ treatment may be effective with icotinib. Fifth, because the adverse reactions with icotinib were less severe than those with gefitinib and erlotinib, especially the occurrence of diarrhea, icotinib can be used for patients who cannot tolerate gefitinib or erlotinib. Zhang et $\mathrm{al}^{46}$ reported one case who switched to icotinib due to grade III-IV diarrhea induced by gefitinib and observed no evident diarrhea thereafter, and the disease was controlled for 7 months with oral administration of icotinib. Sixth, treat other types of carcinoma in addition to lung cancer.

Future registered clinical trials of icotinib were desrcibed in a review article. ${ }^{43}$

\section{First-line routine treatment for patients with EGFR mutations}

The CONVINCE study compares the efficacy of icotinib and combined pemetrexed/cisplatin chemotherapy followed 
by pemetrexed maintenance for treating patients with $E G F R$ mutation-positive advanced NSCLC.

The CTONG-1201 study was designed to evaluate the efficacy and safety of icotinib compared with whole brain radiation therapy in advanced NSCLC patients with brain metastases.

Icotinib is used as a first-line treatment for elderly NSCLC patients with EGFR mutations.

Icotinib is used as an additional treatment for stage IIIA NSCLC with EFGR19/21 exon mutation.

\section{Dose study}

Single-center, single-arm, open-label clinical trials have been initiated to observe the efficacy of icotinib for advanced NSCLC at the dose of $125 \mathrm{mg}$, tid. If the disease progresses, the dose will be increased to $250 \mathrm{mg} / \mathrm{mg}$, tid. If the disease progresses again, the dose will continue to be increased to $375 \mathrm{mg}$, tid. Currently, 23 patients are enrolled, and the results indicate that increasing the dose could benefit the patients.

Patients with advanced or metastasis NSCLC receive icotinib for 8 weeks after progression with chemotherapy. Different dosages of icotinib are administrated to cases who obtained SD, and the efficacy and safety of icotinib as a second-line treatment are being evaluated.

Icotinib is as a first-line therapy for EGFR mutationpositive patients with NSCLC, and cases who present slow progressive disease (PD) receive continuing icotinib or chemotherapy as a second-line therapy. The efficacies between the two groups are being evaluated.

\section{Combined radiotherapy}

ICOME evaluated the safety and tolerability of different doses of icotinib combined with whole brain radiotherapy from NSCLC with brain metastases and mutation-positive of EGFR. The preliminary data suggest that the icotinib concentrations in the cerebrospinal fluid were $2 \%-10 \%$ of the plasma concentration.

\section{For the wild-type}

The efficacy of different doses of icotinib as a second-line treatment for stage IIIB-IV NSCLC with wild-type EGFR is being investigated.

\section{For carcinoma other than lung cancer}

A Phase II study of icotinib as a second-line treatment for esophageal cancer is being conducted.

A Phase I trial in combination with gemcitabine as a firstline treatment for advanced pancreatic cancer and a Phase I/II study for head and neck cancer are being conducted.

\section{Conclusion}

Icotinib hydrochloride was launched a short time ago, only 2 years and 8 months. Long-term survival data have not yet been obtained; therefore, its long-term survival results need to be evaluated for a longer period. Current clinical application of icotinib remains unstandardized, evidence from first-line applications has not been sufficient, and clinical studies with better depth and breadth are needed.

Nevertheless, icotinib is the third worldwide and first Asia-wide, self-developed small-molecule EGFR-TKI inhibitor with completely independent intellectual property rights in the People's Republic of China. Phase I, II, and III clinical trials have confirmed and Phase IV clinical data have further verified its short-term efficacy as a monotherapy for NSCLC. In addition, satisfactory efficacy has been observed in patients with EGFR mutations, and the efficacy is not inferior to gefitinib and erlotinib. ${ }^{47}$ Icotinib has milder adverse reactions, better tolerance, and significantly lower prices compared with gefitinib and erlotinib. Icotinib has now been included in medical insurance in some cities in the People's Republic of China. Upon reaching an efficacy of no less than the SD after 6 months of treatment, complimentary medicine can be offered. Currently, 3,481 patients have received complimentary medicine, and 74,430 boxes of drugs have been distributed. The cumulative drug cost has been over 200 million renminbi (RMB), which reduces the long-term economic burden for Chinese patients with advanced NSCLC. ${ }^{43}$

Icotinib is an important treatment option for patients with advanced NSCLC in the People's Republic of China.

\section{Disclosure}

All of the authors declare no conflicts of interest in this work.

\section{References}

1. Jemal A, Bray F, Center MM, Ferlay J, Ward E, Forman D. Global cancer statistics. CA Cancer J Clin. 2011;61(2):69-90.

2. Han R, Zheng R, Zhang S, Wu M, Chen W. Trend analyses on the differences of lung cancer incidence between gender, area and average age in China during 1989-2008. Zhongguo Fei Ai Za Zhi. 2013;16(9):445-451.

3. Cataldo VD, Gibbons DL, Pérez-Soler R, Quintás-Cardama A. Treatment of non-small-cell lung cancer with erlotinib or gefitinib. $N$ Engl J Med. 2011:364(10):947-955.

4. Wu N, Wang AP, Wang YX. Anti-tumor effects and mechanism of BPI2009, an inhibitor of epidermal growth factor receptor tyrosine. Chin J Pharmacol Ther. 2005;10(4):456-461.

5. Tan F, Zhang L, Zhao Q, et al. Pharmocology and clinical evaluation of icotinib hydrochloride. Chin J New Drugs. 2009;18(18):1-4.

6. Gao Z, Chen W, Zhang X, et al. Icotinib, a potent and specific EGFR tyrosine kinase inhibitor, inhibits growth of squamous cell carcinoma cell line A431 through negatively regulating AKT signaling. Biomed Pharmacother. 2013;67(5):351-356. 
7. Yang G, Yao Y, Zhou J, Zhao Q. Effects of icotinib, a novel epidermal growth factor receptor tyrosine kinase inhibitor, in EGFR-mutated non-small cell lung cancer. Oncol Rep. 2012;27(6):2066-2072.

8. Tan F, Shen X, Wang D, et al. Icotinib (BPI-2009H), a novel EGFR tyrosine kinase inhibitor, displays potent efficacy in preclinical studies. Lung Cancer. 2012;76(2):177-182.

9. Liu D, Jiang J, Zhang L, et al. Clinical pharmacokinetics of Icotinib, an anti-cancer drug: evaluation of dose proportionality, food effect, and tolerability in healthy subjects. Cancer Chemother Pharmacol. 2014;73(4):721-727.

10. Zhao Q, Shentu J, Xu N, et al. Phase I study of icotinib hydrochloride (BPI-2009H), an oral EGFR tyrosine kinase inhibitor, in patients with advanced NSCLC and other solid tumors. Lung Cancer. 2011;73(2): 195-202.

11. Wang HP, Zhang L, Wang YX, et al. Phase I trial of icotinib, a novel epidermal growth factor receptor tyrosine kinase inhibitor, in Chinese patients with non-small cell lung cancer. Chin Med J (Engl). 2011;124(13):1933-1938.

12. Shi $Y$, Zhang L, Liu X, et al. Icotinib versus gefitinib in previously treated advanced non-small-cell lung cancer (ICOGEN): a randomised, double-blind phase 3 non-inferiority trial. Lancet Oncol. 2013;14(10): 953-961.

13. Sun Y. Icotinib in the treatment of the second-line and third-line therapy for patients with advanced non-small cell lung cancer: a prospective multi-center one-arm Phase 3 trial. The 15th annual meeting of Chinese Society of Clinical Oncology (CSCO); 2012; Beijing.

14. Chen P, Li K, Wang CL, et al. Comparison of pharmacoeconomics between icotinib and traditional second-line chemotherapy for patients with advanced non-small cell lung cancer. Shandong Med J. 2013;53(33):47-48.

15. Li R, Peng ZY, Ren SS, Liang H, Sun JD. Observation on efficacy of icotinib hydrochloride for treating advanced non-small cell lung cancer. J Mod Med Health. 2013;29(15):2255-2257.

16. Chen P, Li K, Wang CL, et al. Short-term response and toxicity of icotinib hydrochloride in the treatment for advanced non-small cell lung cancer. J Chin Oncol. 2012;18(12):947-951.

17. Nong JY, Qin N, Wang JH, et al. Clinical analysis for patients with recurrent advanced Non-small cell lung cancer treated with icotinib hydrochloride. Zhongguo Fei Ai Za Zhi. 2013;16(5):240-245.

18. Zhang BB, Lin BC, He CX, et al. A retrospective study of icotinib for patients with brain metastases from non-small cell lung cancer. Chin Clin Oncol. 2013;18(9):786-789.

19. Su XM, Liu Z, Zhu YJ, Liu HY, Zhao ZG, Zhang T. Curative effect observation of icotinib in the treatment of patients with advanced nonsmall cell lung cancer and epidermal growth factor receptor mutations. Chin Gen Pract. 2013;16(11):3967-3969.

20. Shao L, Zhang B, He C, et al. Efficacy and safety of icotinib in Chinese patients with advanced non-small cell lung cancer after failure of chemotherapy. Chin Med J (Engl). 2014;127(2):266-271.

21. Zhang L, Ren GJ. Safety, toleration and therapeutic effect of the new drug icotinib hydrochloride in treating Chinese patients with non-small cell lung cancers. Chin J New Drugs. 2012;21(17):2051-2055.

22. Zhang BB, He CX, Song ZB, et al. Evaluations of advanced adenocarcinoma lung cancer patients with icotinib. J Med Res. 2013;42(11): 61-65.

23. He CX, Zhang BB, Zheng L, et al. Efficacy of icotinib hydrochloride in the treatment of 31 patients with advanced squamous cell lung carcinoma. Chin J New Drugs. 2013;22(12):1430-1434.

24. Chiang CL, Tsai CM, Chou TY, et al. Erlotinib in patients with advanced lung squamous cell carcinoma. Cancer Chemother Pharmacol. 2013;71(1):203-208

25. Hata A, Katakami N, Kunimasa K, et al. Erlotinib for pretreated squamous cell carcinoma of the lung in Japanese patients. Jpn J Clin Oncol. 2011;41(12):1366-1372.

26. Tseng JS, Yang TY, Chen KC, Hsu KH, Chen HY, Chang GC. Retrospective study of erlotinib in patients with advanced squamous lung cancer. Lung Cancer. 2012;77(1):128-133.
27. Song Z, Yu X, Cai J, et al. Efficacy of icotinib for advanced non-small cell lung cancer patients with EGFR status identified. Zhongguo Fei Ai Za Zhi. 2013;16(3):138-143.

28. Gu A, Shi C, Xiong L, Chu T, Pei J, Han B. Efficacy and safety evaluation of icotinib in patients with advanced non-small cell lung cancer. Chin J Cancer Res. 2013;25(1):90-94.

29. Sun JH, Zhang Y, Zhao JB, et al. Analysis of typical cases for patients with advanced non-small cell lung cancer received icotinib. Chin J Clin Oncol. 2012;39(12):868-870.

30. Zeng XM, Hou M, Li ZX. Efficacy of icotinib for advanced lung adenocarcinoma. J Cancer Control Treat. 2013;26(1):12-15.

31. Zhang J, Lu XN, Ban LY. Efficacy and safety of icotinib hydrochloride in the treatment of 50 patients with advanced adenocarcinoma of lung. Chin J New Drugs. 2013;22(9):1068-1071.

32. Sun JH, Zhang Y, Zhao JB, et al. Clinical efficacy of icotinib in treatment of seventeen non-smokers with advanced lung adenocarcinoma. Chin J New Drugs. 2012;21(14):1651-1653.

33. Li X, Yang XJ, Sun YF, et al. Clinical observation of icotinib hydrochloride for patients with advanced non-small cell lung cancer. Chin J Oncol. 2012;34(8):627-631.

34. Yu LQ, Li L, Li J. The clinical efficacy and tolerability of icotinib in 30 advanced non-small cell lung cancer patients. Drug Evaluation. 2012;9(30):24-27.

35. Wu WQ, Lu KH, Zhang ML, Jin SD. Clinical observation of icotinib hydrochloride in the treatment for advanced non-small cell lung cancer. China Cancer. 2013;22(6):497-501.

36. Wang L, Liu JW, Yu PY. Efficacy and toxicity of icotinib hydrochloride in 66 patients with advanced non-small cell lung cancer. Chin J New Drugs. 2013;22(16):1930-1936.

37. Yang X, Zhang H, Qin N, et al. Clinical observation of icotinib hydrochloride in first-line therapy for pulmonary adenocarcinoma. Zhongguo Fei Ai Za Zhi. 2013;16(7):364-368.

38. He Q, Feng ZM, Sun P, et al. Clinical value in treatment with icotinib as first-line therapy in advanced non-small cell lung cancer. J Dalian Medical University. 2013;35(4):365-368.

39. Quan LJ, Mao WM, Xu CQ, Feng YJ. Efficacy observation of 34 patients with advanced non-small cell lung cancer with hydrothorax as the first symptom. Clinical Education General Practice. 2013;11(4): 422-423.

40. Mok TS, Wu YL, Thongprasert S, et al. Gefitinib or carboplatinpaclitaxel in pulmonary adenocarcinoma. NEngl J Med. 2009;361(10): 947-957.

41. Zheng L, Gao LM, Fu ZZ, Kang LY, Dong LX, Bi R. The therapeutic effects observation of icotinib tablets with radiation in patients with advanced stage lung adenocarcinoma. Medical Information. 2013;26(1): $153-154$.

42. Lv C, Ma Y, Feng Q, et al. A pilot study: sequential gemcitabine/ cisplatin and icotinib as induction therapy for stage IIB to IIIA nonsmall-cell lung adenocarcinoma. World J Surg Oncol. 2013;11:96.

43. Sun Y. Targeted treatment with icotinib for NSCLC: evidence from Phase IV trial has been obtained. J Med Res. 2013;42(10):1-2.

44. Li YY, Yu M, Chen FR, Huang MJ. Treatment with high-dose icotinib in a lung adenocarcinoma patient with epidermal growth factor receptor mutation. Chin J New Drugs. 2013;22(11):1307-1310.

45. Guan Y, Zhao H, Meng J, Yan X, Jiao S. Dramatic response to highdose icotinib in a lung adenocarcinoma patient after erlotinib failure. Lung Cancer. 2014;83(2):305-307.

46. Zhang L, Guan QH, Zhao YW, Xia Y, Xiao Y. Successful treatment of one patient with advanced non-small cell lung cancer with icotinib hydrochloride in replacement of gefitinib. Chin J New Drugs. 2013;22(14):1676-1680.

47. Liang W, Wu X, Fang W, et al. Network meta-analysis of erlotinib, gefitinib, afatinib and icotinib in patients with advanced nonsmall-cell lung cancer harboring EGFR mutations. PLoS One. 2014;9(2):e85245. 


\section{Publish your work in this journal}

OncoTargets and Therapy is an international, peer-reviewed, open access journal focusing on the pathological basis of all cancers, potential targets for therapy and treatment protocols employed to improve the management of cancer patients. The journal also focuses on the impact of management programs and new therapeutic agents and protocols on

patient perspectives such as quality of life, adherence and satisfaction. The manuscript management system is completely online and includes a very quick and fair peer-review system, which is all easy to use. Visit http://www.dovepress.com/testimonials.php to read real quotes from published authors.

Submit your manuscript here: http://www.dovepress.com/oncotargets-and-therapy-journal 